\title{
Ferromagneticlike Closure Domains in Ferroelectric Ultrathin Films: First-Principles Simulations
}

\author{
Pablo Aguado-Puente and Javier Junquera \\ CITIMAC, Universidad de Cantabria, Avenida de los Castros s/n, E-39005 Santander, Spain
}

(Received 8 October 2007; published 29 April 2008)

\begin{abstract}
We simulate from first principles the energetic, structural, and electronic properties of ferroelectric domains in ultrathin $\mathrm{SrRuO}_{3} / \mathrm{BaTiO}_{3} / \mathrm{SrRuO}_{3}$ ferroelectric capacitors in short circuit. The domains are stabilized down to two unit cells at zero temperature, adopting the form of a domain of closure, common in ferromagnetic thin films. The domains are closed by the in-plane relaxation of the atoms in the first $\mathrm{SrO}$ layer of the electrode, which behaves more like $\mathrm{SrO}$ in highly polarizable $\mathrm{SrTiO}_{3}$ than in metallic $\mathrm{SrRuO}_{3}$. Even if small, these lateral displacements are very important to stabilize the domains and might provide some hints to explain why some systems break into domains while others remain in a monodomain configuration. An analysis of the electrostatic potential reveals preferential points of pinning for charged defects at the ferroelectric-electrode interface, possibly playing a major role in film fatigue.
\end{abstract}

PACS numbers: 77.80.Dj, 68.55.-a, 77.22.Ej, 77.84.Dy

Ultrathin film ferroelectric capacitors are under active investigation $[1,2]$. Of considerable technological interest as memories, transducers, and electromechanical devices, they present problems of considerable scientific interest. Although technologically relevant films are thicker than $100 \mathrm{~nm}$, a deeper understanding of the origin of these problems requires combined experimental and theoretical studies of thinner regimes. On the one hand, recent breakthroughs in material synthesis and characterization techniques have allowed the growth of ferroelectric thin films with a control at the atomic scale and the local measurement of the ferroelectric properties [3]. On the other hand, the steady increase in computational power and improvements in the efficiency of the algorithms permit accurate first-principles study of larger and more complex systems, overlapping in size with those grown epitaxially.

Prominent among problems of interest is understanding the mechanism screening charge densities at the interfaces. The termination of the ferroelectric polarization at the surface or the electrode interface generates a polarization charge, which gives rise to a depolarizing field tending to suppress the polarization. Two mechanisms are traditionally invoked for the compensation of the polarization charges: the first, screening by charge accumulation at the electrode [4] (or even by ionic adsorbates [5,6]), and the second, the breaking up of the system into domains [7,8].

Previous first-principles local density calculations on realistic short-circuited ferroelectric capacitors suggested that a monodomain configuration for the polarization was unstable below a critical thickness that ranged between $m=2$ and $m=6$ unit cells [9-11] of ferroelectric, depending on the perovskite, the electrode, and the termination at the interface. In all of these approaches, the electrode was the only source of screening, providing free charges that accumulate at the interface on the metallic side and even decay exponentially into the first few layers of the ferroelectric or sharing the ionic displacements responsible for the polarization in the ferroelectric [12]. In any case, the mechanism is ineffective below this critical thickness where the paraelectric phase was stabilized.

In this Letter, we simulate from first principles, within the local density approximation to the density functional theory and the numerical atomic orbital method as implemented in the SIESTA code [13], typical $\mathrm{SrRuO}_{3} / \mathrm{BaTiO}_{3} / \mathrm{SrRuO}_{3}$ ferroelectric capacitors, in which we allow the system to form domains. Our starting point is the reference paraelectric heterostructure described in Ref. [9] that is now replicated $N_{x}$ times along the [100] direction, where $N_{x}$ ranges from 2 to 8 . A soft mode distortion of the bulk tetragonal phase is superimposed on the $\mathrm{BaTiO}_{3}$ layers of the previous paraelectric configuration, so the polarization points upwards in half of the superlattice and downwards in the other half [see inset in Fig. 1(a)]. The twinning on both the $\mathrm{BaO}$ (Ba-centered) and the $\mathrm{TiO}_{2}$ (Ti-centered) planes is considered. Then the atomic positions of all of the ions, both in the electrode and in the ferroelectric thin film, are relaxed until the maximum component of the force on any atom is smaller than $0.01 \mathrm{eV} / \AA$ for $m=2$ and $0.04 \mathrm{eV} / \AA$ for $m=4$. Very accurate computations are required since the differences in energy between relevant phases are 8 orders of magnitude smaller than the absolute value of the energy. The electronic density, Hartree, and exchange-correlation potentials are computed in a uniform real space grid, with an equivalent plane-wave cutoff of $400 \mathrm{Ry}$. We used a $N_{k_{x}} \times$ $12 \times 1$ Monkhorst-Pack mesh for all of the Brillouin zone integrations, where $N_{k_{x}}=\frac{12}{N_{x}}$ except for the interface with $N_{x}=8$, where $N_{k_{x}}=2$. All of the calculations are performed at $T=0$. Details on pseudopotentials and the basis set used can be found in Ref. [14].

Our calculations support stabilization of a polydomain phase with an exceptionally small periodicity below the 

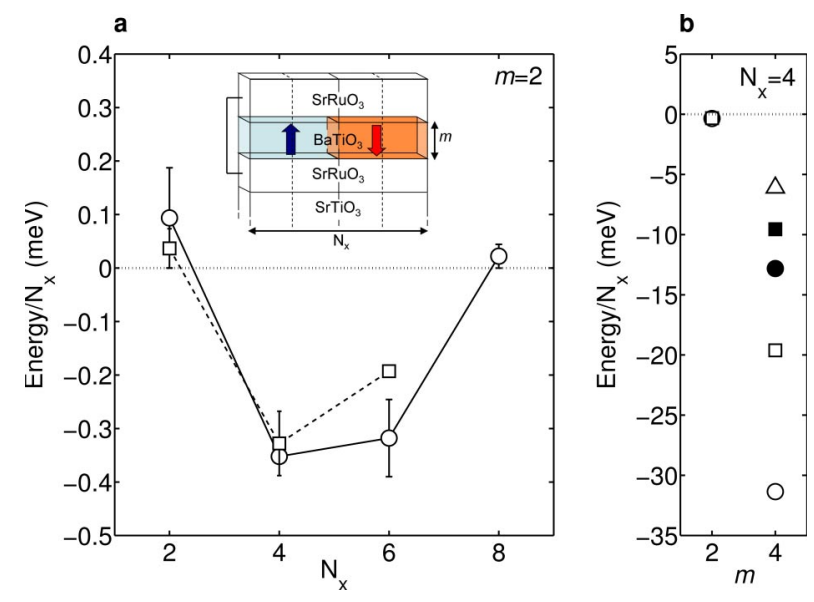

FIG. 1 (color online). Difference in energy between polydomain and paraelectric phases as a function of (a) the domain period $N_{x}$ for a ferroelectric thin film two unit cells thick $(m=2)$ and (b) the thickness of the ferroelectric film for a capacitor with $N_{x}=4$. The energy of the paraelectric phase (dotted line) is taken as a reference. First-principles results for both Ba-centered (circles, solid line) and Ti-centered (squares, dashed) domain walls are shown. In (a), differences in energies between local minima of the polydomain phase are represented by error bars. Inset: Structure of the ferroelectric capacitor considered. $N_{x}$ is the stripe period, and $m$ is the thickness of the ferroelectric thin film, in number of unit cells of the ferroelectric perovskite oxide. In (b), the result for the most stable monodomain configuration is also shown (triangle). Solid symbols correspond to constrained relaxations where no in-plane displacements are allowed.

previous critical thickness [see Fig. 1(a)], in good agreement with the results obtained with Landau theory [15]. For a two-unit-cell-thick film $m=2$, the extra source of screening is efficient provided that the domain period is between 2 and 4 times the thickness of the film. Within this region, the energy cost of forming the domain wall is compensated by reduction of the net polarization charge at the interfaces. As in $180^{\circ}$ stripe domains in bulk [16], the Ba-centered wall configuration is preferred. The energy difference between the most stable polydomain and the paraelectric phase for a capacitor with $m=2$ is very small, of the order of $1.5 \mathrm{meV}(\simeq 16 \mathrm{~K})$ for the whole supercell. For this thickness, there is essentially no energy difference between domains of lateral periods $N_{x}=4$ and 6, suggesting that both might be equally present in a sample. Heating or cooling processes might help the system to overcome potential energy barriers and activate the transition between them. Although the conductive nature of the substrate is different, this fact might provide an extra source of explanation [17] for the intriguing richness in behavior of the stripe domain patterns observed experimentally in $\mathrm{PbTiO}_{3}$ thin films grown on $\mathrm{SrTiO}_{3}$, where two different periods coexisted [8]. (Note that our ratio between domain periods, 1.5 , is close to the experimental factor 1.4 for the so-called $\alpha$ and $\beta$ phases in Ref. [8].)
The energy differences between polydomain and paraelectric phases increase very quickly with thickness [Fig. 1(b)] and amount to 120 (80) meV for a Ba-centered (Ti-centered) domain wall capacitor with $N_{x}=4$ and $m=4$. For this size, the polydomain phases are more stable than the monodomain configuration, itself more stable than the paraelectric phase by $20 \mathrm{meV}$.

The minimum energy structures of these ferroelectric capacitors, shown in Fig. 2, display the closure domain configuration proposed by Landau and Lifshitz [18] and Kittel [19] for magnetic systems. At the center of the $\mathrm{BaTiO}_{3}$ layer, the displacement of the atoms and therefore the corresponding local dipoles point normal to the interface (coordinate $z$ ), as expected for $180^{\circ}$ stripe domains. However, when approaching the ferroelectric-electrode interface, a small tilt towards [100] is observed. Remarkably, the domains are not closed by the surface layer of the ferroelectric [20] but by the in-plane displacements of the $\mathrm{Sr}$ and $\mathrm{O}$ atoms at the first layer of the electrode, which yield a closure domain pattern, with $90^{\circ}$ domain walls with the $z$-oriented domains inside the film (the Sr atom displaces 0.012 and $0.041 \AA$ along $x$ for $m=2$ and $m=4$, respectively). In contrast to the metallic relaxations in monodomain configurations, where ionic displacements penetrate into the metal over a distance of two or three unit cells $[12,21]$, the displacements beyond the second $\mathrm{RuO}_{2}$ layer are negligible, an indication of more

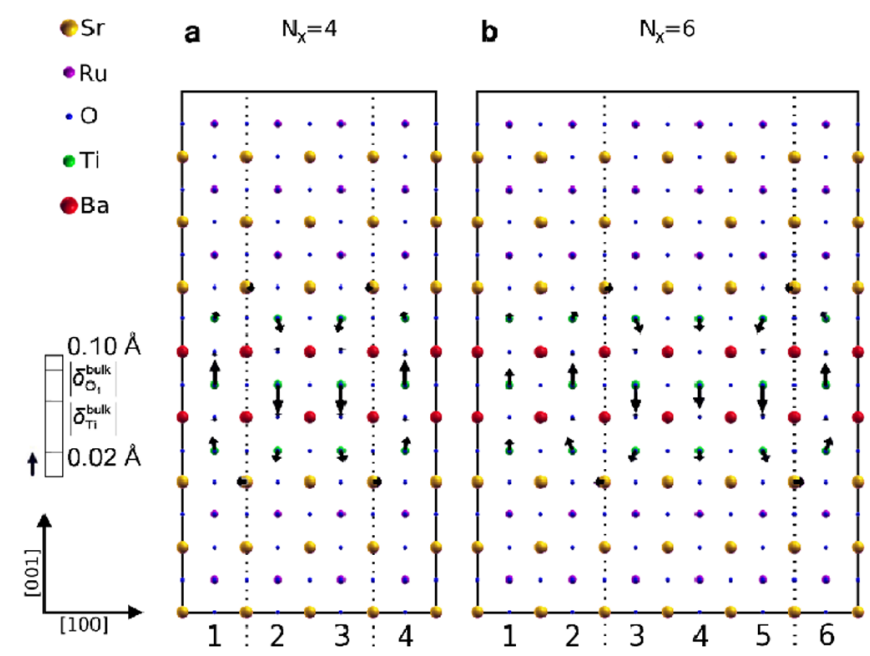

FIG. 2 (color online). Schematic representation of the atomic relaxations in patterns of domains of closure with a domain period of (a) $N_{x}=4$ and (b) $N_{x}=6$. Balls, representing atoms, are located at the positions of the reference paraelectric phase. Atomic displacements for the polydomain configuration after relaxation are represented by arrows, whose magnitude can be gauged with respect to the displacements in the bulk tetragonal phase of $\mathrm{BaTiO}_{3}$ at the scale on the left. Dotted lines indicate the position of the domain wall. Only Ba-centered domains are shown. Similar results are obtained for Ti-centered domains. (For an enlarged figure, see [31].) 
effective screening produced by the domains of closure. The in-plane displacements of the atoms at the interfacial SrO layer, although small in magnitude, stabilize the domain structure. If a constrained relaxation is performed in which the in-plane forces on all of the atoms are artificially eliminated, the atoms move back to the paraelectric positions for $m=2$ or to a structure comparable in energy to the most stable monodomain configuration for $m=4$ [Fig. 1(b)]. Whether the in-plane displacement is allowed or not might partially explain for the very different configurations found experimentally in related heterostructures: Lichtensteiger et al., by using the same experimental setup, have observed how high-quality ultrathin films of $\mathrm{PbTiO}_{3}$ grown on $\mathrm{Nb}-\mathrm{SrTiO}_{3}$ electrodes remain in a monodomain configuration [22] (although with reduced polarization and tetragonality), whereas they form domains when the electrode is replaced by $\mathrm{La}_{0.67} \mathrm{Sr}_{0.33} \mathrm{MnO}_{3}$ [23]. The same domain formation is suggested for $\mathrm{Pb}\left(\mathrm{Zr}_{0.2} \mathrm{Ti}_{0.8}\right) \mathrm{O}_{3}$ on $\mathrm{SrRuO}_{3}$ [24].

Regarding the origin of this polarization-induced relaxation, the analysis of the projected density of states (not shown here) shows that the $\mathrm{SrO}$ layer closest to the interface behaves more like $\mathrm{SrO}$ in $\mathrm{SrTiO}_{3}$ than $\mathrm{SrO}$ in metallic $\mathrm{SrRuO}_{3}$. Similar behavior was found in $A \mathrm{O} / A \mathrm{TiO}_{3}$ heterostructures [14], where $A=\mathrm{Ba}$ or Sr. Both first-principles computations [25] and experimental measurements [26] have shown that $\mathrm{SrTiO}_{3}$ is highly polarizable when combined with $\mathrm{BaTiO}_{3}$ in heterostructures.

Similar domain patterns have been found by using a first-principles effective Hamiltonian for $\mathrm{Pb}\left(\mathrm{Zr}_{0.4} \mathrm{Ti}_{0.6}\right) \mathrm{O}_{3}$ [17] asymmetrically screened (grown on a nonconducting substrate and with a metal with a dead layer as the top electrode) and by using a Landau-Ginzburg phenomenological approach for a $\mathrm{PbTiO}_{3}$ thin film [27], both asymmetrically and symmetrically coated with insulating $\mathrm{SrTiO}_{3}$. Here the domains of closure are obtained even for a symmetrical metal $/ \mathrm{BaTiO}_{3} /$ metal capacitor, with an uniaxial ferroelectric that profoundly dislikes rotating the polarization and in-plane dipoles [20] and where the metallic plates should provide significant screening.

The polarization can be estimated from the structural calculations. Figure 3 displays how much the polar distortion along $z$ is changed by the presence of a domain pattern. We define as $\Delta$ the average of the change of distance, with respect to the most stable paraelectric configuration, between a Ti atom and the nearest $\mathrm{O}$ atom lying on top along the $z$ direction (cf. Ref. [16]), normalized with respect to the short Ti-O distance in the tetragonal bulk phase. $\Delta_{\text {norm }}$ is a very sensitive indicator of the polar order: It is zero as long as the atoms lie in the paraelectric position and tends to unity as the full bulk polar distortion is attained. Figure 3 shows a very narrow $180^{\circ}$ domain wall, about a lattice constant wide, across which the polar distortion symmetrically reverses its sign. In contrast to $180^{\circ}$ domains in bulk [16], where the ferroelectric distor-
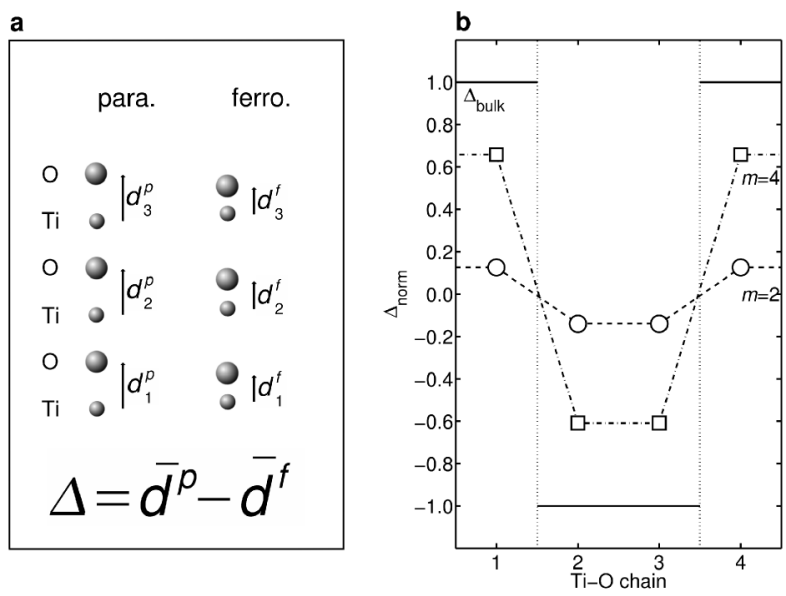

FIG. 3. Measurement of the polarization in the ferroelectric layer as a function of position along the [100] direction of the capacitor. (a) Definition of the average change in distance $\Delta$ between $\mathrm{Ti}$ and apical $\mathrm{O}$ in a chain along [001] for an interface with $m=2$. In every case, the atomic positions correspond to the lowest energy structure. A positive value of $\Delta$ means a polarization pointing upward. (b) Profile of the normalized averaged change in distance along $z$ as a function of the position of the chain for a Ba-centered interface of domain period $N_{x}=4$. The chains are numbered as indicated in Fig. 2. Results are shown for $m=2$ (circles, dashed line) and $m=4$ (squares, dotted-dashed line). Dotted lines represent the position of the domain walls.

tion fully recovers its bulk value by the second atomic plane far away from the domain wall, here it amounts only to $13 \%$ of the bulk value at the center of each domain for a $m=2$ structure, suggesting that the polarization for the thin film is 1 order of magnitude smaller than in bulk. This mean polarization increases with thickness and already amounts to $60 \%$ for a thin film four unit cells thick $(m=4)$.

Ideally, closure domains do not produce any polarization charge since the normal component of the polarization is preserved across any domain wall. Therefore, the depolarizing field should vanish everywhere [19], and a constant electrostatic potential is expected. To further check this point, we plot in Fig. 4 the nanosmoothed $[28,29]$ electrostatic potential along $z$ as a function of the position along the [100] direction of the capacitor. No nanosmoothing is performed along $x$. For a stripe of thickness $m=2$ and period $N_{x}=4$, the potential is essentially flat at the center of the domain, in contrast to the depolarizing field reported for monodomain configurations [9]. A large microscopic field along [100] appears inside the domains of closure at the metal-ferroelectric interface. The origin of this field is due to the difference in polarization in the domain of closure and inside the thin film in our realistic capacitor. Besides, after nanosmoothing in $z$, a residual depolarizing field along [001] is identified in the neighborhood of the domain wall, decaying rapidly away from it. This last field might be responsible for the lowering of the polarization 


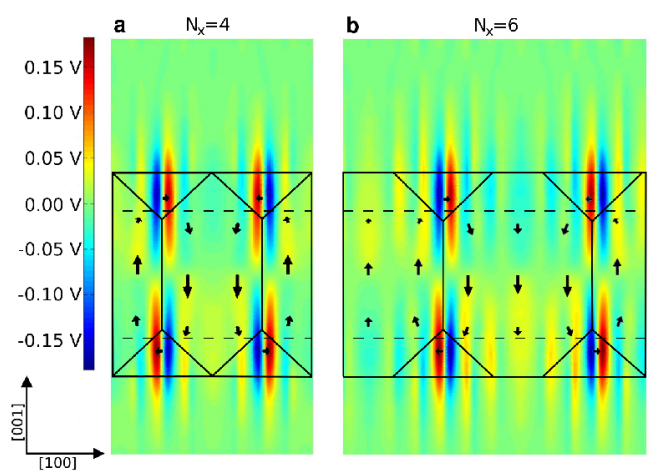

FIG. 4 (color online). Map of the nanosmoothed electrostatic potential in a two-unit-cell-thick ferroelectric capacitor with a stripe period of (a) $N_{x}=4$ and (b) $N_{x}=6$. The arrows represent the atomic displacements with respect to the paraelectric phase as in Fig. 2. Only the displacements of the cations are shown for simplicity. Solid lines are a schematic representation of the domains of closure, while dashed lines mark the position of the $\mathrm{BaTiO}_{3} / \mathrm{SrRuO}_{3}$ interface.

with respect to bulk shown in Fig. 3. Both fields might play an important role in the fatigue of ferroelectric capacitors, the most serious device problem in ferroelectric thin films [1]. In particular, we identify at the ferroelectric-electrode interface the preferred points of migration of charged defects, which pin the domain walls and inhibit their motion [30]. The depolarizing field at the center of the domain increases with the domain period; it starts to be appreciable for $N_{x}=6$ [Fig. 4(b)] and finally destabilizes the ferroelectric distortions for $N_{x}=8$, as shown in Fig. 1(a).

Although we have demonstrated that the domains are stable, it is not clear whether the capacitor as a whole can be called ferroelectric since, for this, the polarization has to be switchable under external electric fields $[20,24]$.

Our calculations provide insightful results on the energetic, structural, and electronic properties of ferromagneticlike closure domains in ultrathin ferroelectric capacitors. We provide some hints to explain why some systems break into domains while others remain in a monodomain configuration. We also predict the preferential sites for pinning charged defects, important for understanding the fatigue of thin films.

We thank M.H. Cohen for the critical reading of the manuscript and J.F. Scott for useful discussions. Calculations were performed on the computers of the ATC group and on the Altamira Supercomputer at the Universidad de Cantabria. This work was supported by the Spanish MEC under Projects No. FIS2006-02261 and No. FPU AP2006-02958 and by the Australian Research Council ARC Discovery Grant No. DP 0666231.
[1] M. Dawber, K. M. Rabe, and J. F. Scott, Rev. Mod. Phys. 77, 1083 (2005).

[2] Ph. Ghosez and J. Junquera, Handbook of Theoretical and Computational Nanotechnology (American Scientific, Stevenson Ranch, CA, 2006), Vol. 9, pp. 623-728.

[3] C. H. Ahn, K. M. Rabe, and J.-M. Triscone, Science 303, 488 (2004).

[4] R. R. Mehta, B. D. Silverman, and J. T. Jacobs, J. Appl. Phys. 44, 3379 (1973).

[5] D. D. Fong et al., Phys. Rev. Lett. 96, 127601 (2006).

[6] J. E. Spanier et al., Nano Lett. 6, 735 (2006).

[7] S. K. Streiffer et al., Phys. Rev. Lett. 89, 067601 (2002).

[8] D. D. Fong et al., Science 304, 1650 (2004).

[9] J. Junquera and Ph. Ghosez, Nature (London) 422, 506 (2003).

[10] Y. Umeno, B. Meyer, C. Elsässer, and P. Gumbsch, Phys. Rev. B 74, 060101(R) (2006).

[11] C.-G. Duan, R. F. Sabirianov, W.-N. Mei, S. S. Jaswal, and E. Y. Tsymbal, Nano Lett. 6, 483 (2006).

[12] G. Gerra, A. K. Tagantsev, N. Setter, and K. Parlinski, Phys. Rev. Lett. 96, 107603 (2006).

[13] J. M. Soler et al., J. Phys. Condens. Matter 14, 2745 (2002).

[14] J. Junquera, M. Zimmer, P. Ordejón, and Ph. Ghosez, Phys. Rev. B 67, 155327 (2003).

[15] A. M. Bratkovsky and A. P. Levanyuk, Integr. Ferroelectr. 84, 3 (2006); Appl. Phys. Lett. 89, 253108 (2006).

[16] B. Meyer and D. Vanderbilt, Phys. Rev. B 65, 104111 (2002).

[17] S. Prosandeev and L. Bellaiche, Phys. Rev. B 75, 172109 (2007).

[18] L. Landau and E. Lifshitz, Phys. Z. Sowjetunion 8, 153 (1935).

[19] C. Kittel, Phys. Rev. 70, 965 (1946).

[20] B.-K. Lai et al., Phys. Rev. Lett. 96, 137602 (2006); Phys. Rev. B 75, 085412 (2007).

[21] M. Stengel and N. A. Spaldin, Nature (London) 443, 679 (2006).

[22] C. Lichtensteiger, J.-M. Triscone, J. Junquera, and Ph. Ghosez, Phys. Rev. Lett. 94, 047603 (2005).

[23] C. Lichtensteiger et al., Appl. Phys. Lett. 90, 052907 (2007).

[24] V. Nagarajan et al., J. Appl. Phys. 100, 051609 (2006).

[25] J. B. Neaton and K. M. Rabe, Appl. Phys. Lett. 82, 1586 (2003).

[26] W. Tian et al., Appl. Phys. Lett. 89, 092905 (2006).

[27] G. B. Stephenson and K. R. Elder, J. Appl. Phys. 100, 051601 (2006).

[28] A. Baldereschi, S. Baroni, and R. Resta, Phys. Rev. Lett. 61, 734 (1988).

[29] J. Junquera, M. H. Cohen, and K. M. Rabe, J. Phys. Condens. Matter 19, 213203 (2007).

[30] L. He and D. Vanderbilt, Phys. Rev. B 68, 134103 (2003).

[31] See http://personales.unican.es/junqueraj/downloadfigure2.pdf. 